\title{
Ankle Orthosis-induced Decrease in Repetitive Rebound Jump Height: Relationship With Restriction in Sagittal Ankle Range of Motion
}

\author{
Morikawa Masanori \\ Hiroshima University \\ Maeda Noriaki \\ Hiroshima University \\ Komiya Makoto \\ Hiroshima University \\ Kobayashi Toshiki \\ The Hong Kong Polytechnic University \\ Urabe Yukio ( $\nabla$ yurabe@hiroshima-u.ac.jp ) \\ Hiroshima University
}

\section{Research Article}

Keywords: ankle lateral ligament, sports performance, ankle brace, ankle support, ankle orthotics

Posted Date: September 27th, 2021

DOI: https://doi.org/10.21203/rs.3.rs-892632/v1

License: (9) This work is licensed under a Creative Commons Attribution 4.0 International License. Read Full License 


\section{Abstract}

Background: Ankle orthotics decreases the maximal vertical jump height. It is essential to maximize jump height and minimize ground contact time during athletic performance. However, the effect of ankle orthotics on athletic performance has not been reported. We aimed to investigate the effect of ankle orthotics on squat jump (SJ), countermovement jump (CMJ), and repetitive rebound jump (RJ) performance and the relationship between jump performance and restriction in sagittal ankle range of motion.

Methods: Twenty healthy volunteers performed SJ, CMJ, repetitive RJ under no-orthosis and two orthotic conditions (orthosis 1 and orthosis 2). During SJ and CMJ, we measured the vertical ground reaction force and calculated the following parameters: jump height, peak vertical ground reaction force, rate of force development, net vertical impulse, and peak power. During repetitive RJ, the jump height, contact time, and RJ index were measured. A two-dimensional motion analysis was used to quantify the ankle range of motion in the sagittal plane during $\mathrm{SJ}, \mathrm{CMJ}$, and repetitive $\mathrm{RJ}$.

Results: Multivariate analysis of variance and the post hoc test showed a significant decrease in the vertical jump height $(p=0.003)$, peak power $(p=0.007)$, and maximum plantarflexion and dorsiflexion angles ( $p<0.001)$ during SJ using orthosis 2 compared to those using the no-orthosis condition. Additionally, orthosis 2 significantly decreased the jump height at the end of repetitive RJ ( $p=0.046)$, during which a significant negative correlation was found between jump height and maximum dorsiflexion angle $(r=0.485, p=0.030)$.

Conclusions: An ankle orthosis-induced restriction of dorsiflexion is associated with a reduction in jump height during static jump and repetitive RJ performance.

\section{Background}

Ankle orthotics is a common measure for preventing ankle sprain. Meta-analyses have shown that ankle orthotics is effective for the primary and secondary prevention of acute ankle injuries among athletes [1]. Nevertheless, ankle orthotics may have a negative impact on athlete performance in activities such as sprinting [2], cutting [3], jump landing [4,5], and vertical jumping [2]. Furthermore, there is a consensus that ankle orthotics reduces the vertical jump height [6-12]. This may be due to a decrease in the ankle range of motion (ROM) in the sagittal plane $[10,11]$, rectus femoris and calf muscle activity $[8,9,11]$, or vertical ground reaction force (VGRF) [8,12].

However, since the ankle orthosis-related decrease in jump height is small $(0.013 \mathrm{~m})$, it is controversial whether ankle orthotics influences vertical jump performance during practice or competition. Moreover, the influence of ankle orthotics on jump performance varies depending on the sport type and competition level [10]. Most previous studies focused on the maximum jump height for static vertical jumps such as squat jump (SJ) [8-10] or countermovement jump (CMJ) [6-8,11]; hence, some studies [6,12] pointed out the difference between the experimental situation and the actual field situation. Certainly, the 
maximum jump height is an essential factor for athletic performance. Nonetheless, when a basketball player jumps to get a rebound ball or a volleyball player jumps to block a spiked ball, they repetitively jump vertically to maximize jump height and minimize the time taken to attain the maximum point. Consequently, based on results obtained during the $\mathrm{SJ}$ and $\mathrm{CMJ}$, there is a limitation in concluding the impact of ankle orthosis use on the jumping performance. Therefore, the measurement of parameters other than jump height during more realistic jump movements is required.

Reactive strength may be used to assess the athlete's ability to attain maximum jump height and minimum ground contact time during repetitive rebound jump (RJ) [13]. It also indicates an athlete's ability to rapidly generate force under a high eccentric load [14]. Jump height, ground contact time, and reactive jump index (ratio of jump height and ground contact time) were used as parameters in previous studies to evaluate jump performance in the sports field [15]. However, no study has investigated whether the use of ankle orthotics decreases repetitive RJ performance.

Therefore, we aimed to investigate the influence of ankle orthotics on SJ, CMJ, and repetitive RJ performance, and the relationship between jump performance and dorsi-plantarflexion ROM in healthy adults.

\section{Methods}

\section{Participants}

Twenty recreationally active volunteers ( 15 men and 5 women) agreed to participate in the study (mean \pm standard deviation [SD] of age, body height, weight, and body mass index: $23.9 \pm 2.5$ years, $168.4 \pm 8.41$ $\mathrm{cm}, 61.8 \pm 12.5 \mathrm{~kg}$, and $21.6 \pm 3.1 \mathrm{~kg} / \mathrm{m}^{2}$, respectively) after being informed about this study protocol. The study protocol was approved by the Ethics Committee for epidemiology of XXX University (approval number: E-2268). All subjects provided informed consent for their participation in the study. "Recreationally active" was defined as participation in at least 150 min of moderate activity per week for at least 6 months prior to the study [16]. Participants were experienced in athletics, basketball, baseball, classical ballet, badminton, football, tennis, swimming, or volleyball. We excluded participants with lower extremity injury and symptoms, previous lower extremity surgery prior to the study, vestibular disease, or neurological impairments.

\section{Study design and procedures}

This study used a laboratory-based and repeated-measures test design. To determine the influence of ankle orthotics on jump performance and dorsi-plantarflexion ROM, we used the following experimental protocol with participants in a barefoot condition (no-orthosis) and two orthotics conditions (orthosis 1 and orthosis 2) with different restrictions on dorsi-plantarflexion. Filmista (Nippon Sigmax, Japan) and A1 (Nippon Sigmax, Japan) orthotics were used for orthosis 1 and 2 conditions, respectively. Participants wore correctly sized orthotics on both ankles. A certified orthotist instructed participants on how to wear the orthotics using demonstrations. Figure 1 shows the composition of the orthotics used in this study. 
Orthosis 1 consists of three thin layers (Figure 1A). It has two surfaces made of soft and hard urethane films with different elasticities. The hard film is found in the middle layer and is designed to limit excessive ankle inversion without restricting the ROM of the ankle joint in the sagittal plane. Orthosis 2 consists of three different straps on the fabric, covering the ankle joint with medial and lateral stays (Figure 1B). Stirrup, biceps, and distal tibiofibular joint straps are applied to prevent excessive ankle inversion, based on the medical taping concept. These ankle orthotics were made by the same manufacturer; however, orthosis 1 had less restriction on ankle inversion than orthosis 2. Participants successively repeated the same jump exercises under the three abovementioned conditions following a randomized order. A jump session was defined as a period during which a participant performed static jumps and RJ under one of the above conditions. All parameters were measured over a 3-day period for each participant. Participants completed a jump session under one condition per day, with a minimum of $24 \mathrm{~h}$ of rest between each jump session.

\section{Vertical jump performance tests}

Participants performed a 5-min warm-up exercise before undergoing the vertical jump performance tests. They received explanations on how to perform SJ, CMJ, and RJ and practiced these vertical jumps. To perform the SJ, they started by folding their hands and squatting with their hips and knees flexed to approximately 45 and their feet placed a shoulder width apart on a force plate (Technology Service, Nagano, Japan). After 1-2 s, the examiner instructed the participants to jump vertically and forcefully. To perform the $\mathrm{CMJ}$, they began by folding their hands and standing upright with their feet placed approximately a shoulder width apart on the force plate. After $1-2 \mathrm{~s}$, they rapidly descended into a 45 semi-squat position and jumped vertically with maximum effort. Five sets, each of $\mathrm{SJ}$ and $\mathrm{CMJ}$, were performed. Participants took as many breaks as needed between each set to avoid fatigue.

The repetitive RJ performance was assessed using the Optojump ${ }^{\mathrm{TM}}$ system (Microgate, Bolzano, Italy), consisting of two infrared photocell bars with one bar acting as a transmitter unit containing 96 lightemitting diodes positioned $3 \mathrm{~mm}$ above the ground, and the other bar acting as a receiver unit.

Participants were instructed to keep their hands on their hips to avoid upper-body interference, jump, and land on the same spot, with legs extended then flexed, while looking ahead. They were also instructed to maximize the jump height and minimize the ground contact time. In a previous study, this method of RJ assessment achieved interday reliability [17]. When a participant performed the repetitive RJ within a parallel bar configuration, the light from the light-emitting diode was interrupted by the participant's foot during the jump, triggering the timer in the unit and recording the interruption with a sampling frequency of $1000 \mathrm{~Hz}$. Two sets of seven repetitive RJs were performed with intermittent 5-min resting periods.

\section{Analysis of the vertical jump performance}

SJ and CMJ performances were analyzed using the VGRF, recorded by a force plate with a sampling frequency of $1000 \mathrm{~Hz}$. Based on previous studies [18], jump height [cm], rate of force development (RFD) $[\mathrm{N} / \mathrm{s}]$, vertical impulse [Ns], peak power [W], and maximum VGRF (VGRFmax) [N] were calculated using 
MATLAB (R2020b, Math Works GK, Tokyo, Japan). The jump height was estimated as follows: jump height $=(1 / 2 \times \text { Tair } \times g)^{2} \times(2 g)^{-1}$. Tair represents the flight time [s] from the force record on the force plate, and $g$ the acceleration due to gravity $\left(9.81 \mathrm{~m} / \mathrm{s}^{2}\right)$. The RFD was calculated as: RFD $=($ VGRFmax minimum VGRF [VGRFmin]) / $\Delta \mathrm{t} 1$, where $\Delta \mathrm{t} 1$ indicates the change in time [s] between $20 \%$ and $80 \%$ of the total time from the VGRFmin to the VGRFmax. VGRFmin was defined as the lowest value of the VGRF during the contact phase before increasing to VGRFmax. VGRFmax was defined as the peak of the VGRF occurring for the first time if two peaks were applicable. The net vertical impulse was calculated as VGRF $\times \Delta \mathrm{t} 2$ - body weight $\times \Delta \mathrm{t} 2$, where $\Delta 2$ indicates the change in time [s] from the point at which the VGRF equated with the body weight to the point at which VGRF fell below the body weight. Peak mechanical power was calculated from the vertical jump height and body weight as $(60.7 \times$ jump height $[\mathrm{cm}])+(45.3$ $x$ body height $[\mathrm{cm}])$. The RFD and VGRFmax were normalized using the body weight $[\mathrm{kg}]$ to calculate the relative RFD and relative VGRFmax.

Optojump ${ }^{\mathrm{TM}}$ proprietary software (Optojump ${ }^{\mathrm{TM}}$ Next software, version 1.9.9.0, Bolzano, Italy) was used to automatically calculate RJ performance variables (jump height [cm], contact time [s], and RJ index (RJ index) $[\mathrm{m} / \mathrm{s}]$ ). The RJ index was estimated as: $\mathrm{RJ}$ index $=1 / 8 \times \mathrm{g} \times \mathrm{Tair}^{2} /$ contact time. Of the seven repetitive jumps in the second set, the second, third, fourth, and fifth jumps were included in the analysis. The individual performance variables per RJ (second, third, fourth, fifth, and sixth jumps) and their means were used for further statistical analysis.

\section{Analysis of the sagittal ankle joint motion}

Two-dimensional motion analysis for sagittal ankle joint motion was performed simultaneously with the vertical performance test parameters ( $\mathrm{SJ}, \mathrm{CMJ}$, and $\mathrm{RJ})$. Three reflective markers were placed on the dominant leg on the lateral aspect of the tibial plateau, lateral malleolus, and lateral aspect of the base of the fifth metatarsal [10]. The markers were applied by the same examiner. A video camera was positioned at a distance of $1.5 \mathrm{~m}$ perpendicular to the edge of the force plate or OptoJump ${ }^{\mathrm{TM}}$ device to capture the trajectory of the marker from the sagittal plane with a sampling frequency of $240 \mathrm{~Hz}$. Image J software (National Institutes of Health, Maryland, USA) was used for the analysis. The maximum dorsiflexion angle, plantarflexion angle at toe-off, and dorsi-plantarflexion ROM were calculated by connecting three points [10], as shown in Figure 2. The ROM was defined as the difference between the plantarflexion angle at toe-off and the maximum dorsiflexion angle. For each joint angle, the mean angle from the five jumps was used as the representative value. Additionally, the individual angle of the second set of RJ was used for further analysis.

\section{Statistical analysis}

A one-factor (type of orthosis: no-orthosis, orthosis 1, orthosis 2) repeated-measures multivariate analysis of variance (MANOVA) was used to determine the influence of ankle orthotics on the variables of jump performance and ankle ROM. MANOVA was conducted for each type of jump (SJ, CMJ, RJ). A univariate 
analysis of variance (ANOVA) and Tukey method for pairwise comparisons were conducted on any significant findings.

To determine the difference in the effect of ankle orthosis use on each RJ-related variable (jump height, contact time, and RJ index) and the angle for sagittal ankle motion by the number of RJs, a two-factor (type of orthosis: no-orthosis, orthosis 1, orthosis $2 \times$ number of RJ: second, third, fourth, fifth, and sixth) repeated-measures MANOVA was initially conducted. As a follow-up analysis, a two-factor ANOVA was conducted on any significant findings. If the main or interaction effect was observed, the Tukey method of pairwise comparisons was also performed. Additionally, to explore the relationship between the RJrelated variables and the angle of sagittal ankle motion during RJs, a Pearson's product moment correlation coefficient was computed for the variables that were significant by pairwise comparisons. A statistical significance was defined as $p<0.05$ in all statistical tests used in this study.

\section{Results}

Table 1 summarizes the mean \pm SD of the measurement variables in this study and their levels of statistical significance. MANOVA showed a significant difference in the vertical jump performance only during SJ performance $(p=0.021)$ under orthotic conditions; the vertical jump performances were not significantly different for participants who performed CMJ $(p=0.118)$ and RJ $(p=0.391)$. The mean jump height, peak power, and angle of sagittal ankle motion during SJ performance systematically decreased in the following order: no-orthosis, orthosis 1 , and orthosis 2 conditions. Pairwise comparisons after univariate analysis showed a significant decrease in the vertical jump height $(p=0.003)$, peak power $(p=0.007)$, and sagittal ankle ROM ( $p<0.001)$ during SJ performance using orthosis 2 compared to the same parameters measured under no-orthosis conditions.

Figure 3 shows the temporal changes in jump height, contact time, and RJ index during repetitive RJ, depending on the orthosis condition. The jump height significantly decreased only at the sixth RJ under the orthosis 2 condition compared to that under the no-orthosis condition. At the sixth RJ, as shown in Figure 4, the angle of maximum dorsiflexion, plantarflexion at toe-off, and their ROMs were restricted under the orthosis 2 condition, which was a significant change compared to that under the no-orthosis condition $(p<0.001)$. Figure 5 presents the relationship between jump height and sagittal ankle ROM at the sixth RJ based under the orthotics condition. A significant negative correlation was found between jump height and maximum dorsiflexion angle under the orthosis 2 condition $(r=-0.485, p=0.030)$.

\section{Discussion}

This study aimed to investigate the effect of ankle orthotics with different degrees of restriction of the ankle ROM in the sagittal plane, particularly on repetitive RJ performance. A significant relationship between dorsiflexion angle and a decrease in jump height was observed only in the orthosis 2 condition, which had the higher restriction on the ankle joint. This suggests that a large ankle orthosis-induced restriction in the ankle ROM results in decreases in the jump height during repetitive RJ. 
The repetitive jump heights increased with the number of jumps under the no-orthosis condition, but not under orthotic conditions; the difference in jump height was significant at the end of RJ. A previous study reported that the stretch-shortening cycle, enabling the production of high muscle forces and elastic energy storage [19], is a key muscle ability during repetitive RJ [20] because it allows for an explosive release of stored energy for subsequent jumps. Thus, ankle orthotics may interfere with this normal function during repetitive RJ. The sagittal ankle ROM was significantly restricted in the orthosis 2 condition during repetitive RJ. Additionally, the dorsiflexion ROM was significantly correlated with jump height only in the orthosis 2 condition. Importantly, maximum dorsiflexion occurs during the landing phase, which coincides with the critical period of force storage and may affect the energy released for the following jump phase. Therefore, this study indicated that the use of an ankle orthosis decreases repetitive RJ performance due to restricted ankle ROM in the sagittal plane.

The jump height, peak power, and ankle ROM were systematically decreased in SJ in the order of noorthosis, orthosis 1 , and orthosis 2 conditions. Moreover, a significant difference was recorded in these parameters between the orthosis 2 and no-orthosis condition. Mann et al. [10] reported that two ankle orthotics with similar restrictive functions decreased jump height and ankle ROM in the sagittal plane during SJ; these parameters did not significantly change under the no-orthosis condition in this previous study. Another study found a decrease in peak power and jump height during SJ [21]. These studies indicate that restricted ankle ROM in the sagittal plane caused by ankle orthotics is associated with reduced jump height as a result of decreased peak power; these results were corroborated by our study findings. In contrast, the ankle orthotics did not significantly decrease jump height in CMJ and RJ. Although ankle orthotics tended to decrease jump height, the negative effect of ankle orthotics may relatively decrease during complex movements. However, additional research including a more detailed examination of kinematics and kinetics is warranted.

The present study has some limitations. First, it is difficult to generalize our results to other types of ankle orthotics. In addition to the soft ankle orthosis used in this study, a semi-rigid ankle orthosis may limit joint motion by a greater degree. However, the semi-rigid ankle orthosis was not used in this study, and it is not possible to present the effect of the type of ankle orthosis on repetitive RJ performance. Second, we did not recruit athletes with ankle sprains or chronic ankle instability; thus, further studies including these athletes may be required, although ankle orthosis is also used for the primary prevention of ankle sprains.

\section{Conclusions}

This study showed that the orthosis 2 condition, restricting the ankle ROM in the sagittal plane more than the no-orthosis and orthosis 1 conditions, decreased the vertical jump height during jump from static positions (SJ and $\mathrm{CMJ}$ ). Additionally, during repetitive RJ, the jump height increased with the number of jump times in the no-orthosis condition, but not in the orthotic conditions. Moreover, the final jump height significantly decreased in the orthosis 2 condition compared to that in the no-orthosis condition. Finally, under the orthosis 2 condition, a correlation was observed between the final jump height and maximum 
dorsiflexion angle during repetitive RJ, indicating that an ankle orthosis with a large ROM restriction may reduce repetitive $\mathrm{RJ}$ performance.

\section{Abbreviations}

ANOVA, analysis of variance; CMJ, countermovement jump; MANOVA, multivariate analysis of variance; RFD, rate of force development; RJ, rebound jump; RJ index, RJ index; ROM, range of motion; SD, standard deviation; SJ, squat jump; VGRF, vertical ground reaction force; VGRFmax, maximum VGRF; VGRFmin, minimum VGRF

\section{Declarations}

\section{Ethics approval and consent to participate}

The study protocol was approved by the Ethics Committee for epidemiology of Hiroshima University (approval number: E-2268). All subjects provided informed consent for their participation in the study.

\section{Consent for publication}

Not applicable.

\section{Availability of data and materials}

If requested, it will be available.

\section{Competing Interests}

None of the authors has any conflicts of interest associated with this study.

\section{Funding}

The authors have no financial affiliations and/or involvement with any commercial organizations.

\section{Authors' contributions}

$\mathrm{KM}, \mathrm{NA}$ and $\mathrm{YU}$ designed the project and aided in interpreting the results; MM

directed the project, developed the theoretical framework, performed the analysis, and drafted the manuscript and designed the figures. TK edited the manuscript. All authors provided critical feedback and helped shape the research, analysis and manuscript. All authors read and approved the final manuscript.

\section{Acknowledgements}

Not applicable 


\section{Authors' information}

Masanori MORIKAWA

Employment: 1) Graduate student, Department of Sport Rehabilitation, Graduate School of Biomedical and Health Sciences, Hiroshima University, Hiroshima, Japan

2) Research assistant, Department of Preventive Gerontology, Center for Gerontology and Social Science, National Center for Geriatrics and Gerontology, Aichi, Japan

Degree: MSc

Research interests: Effect of orthosis on injury prevention and sports performance.

E-mail: masanori-m@hiroshima-u.ac.jp

Noriaki MAEDA

Employment: Lecturer, Department of Sport Rehabilitation, Graduate School of Biomedical and Health Sciences, Hiroshima University, Hiroshima, Japan

Degree: PhD

Research interests: Evaluation of physical performance and physical conditioning

E-mail: norimmi@hiroshima-u.ac.jp

Makoto Komiya

Employment: Assistant Professor, Department of Sport Rehabilitation, Graduate School of Biomedical and Health Sciences, Hiroshima University, Hiroshima, Japan

Degree: PhD

Research interests: Injury prevention, rehabilitation and conditioning, disabled sports

E-mail: makoto-komiya@hiroshima-u.ac.jp

Toshiki Kobayashi

Employment: Associate Professor, Department of Biomedical Engineering, Faculty of Engineering, The Hong Kong Polytechnic University, Hong Kong, China

Degree: PhD

Research interests: Gait biomechanics, prosthetics, orthotics, assistive technologies, Movement disorders 
E-mail: toshiki.kobayashi@polyu.edu.hk

Yukio Urabe

Employment: Prof., Department of Sport Rehabilitation, Graduate School of Biomedical and Health Sciences, Hiroshima University, Hiroshima, Japan

Degree: $\mathrm{PhD}$

Research interest: ACL injury and prevention, and sports biomechanics

E-mail: yurabe@hiroshima-u.ac.jp

\section{References}

1. Barelds I, van den Broek AG, Huisstede B. Ankle bracing is effective for primary and secondary prevention of acute ankle injuries in athletes: a systematic review and meta-analyses. Sports Med. 2018;48:2775-84.

2. Newman TM, Vairo GL, Buckley, WE. The comparative effects of ankle bracing on functional performance. J Sport Rehabil. 2018;27:491-502.

3. Lee WC, Kobayashi T, Choy BT, Leung AK. Comparison of custom-moulded ankle brace with hinged joints and off-the-shelf ankle braces in preventing ankle sprain in lateral cutting movements. Prosthet Orthot Int. 2012;36:190-5.

4. Maeda N, Urabe Y, Tsutsumi S, Numano S, Morita M, Takeuchi T, et al. Effect of semi-rigid and soft ankle braces on static and dynamic postural stability in young male adults. J Sports Sci Med. 2016;15:352-7.

5. Maeda N, Yukio U, Sasadai J, Shuhei N. Effect of soft and semi-rigid ankle braces on kinematic and kinetic changes of the knee and ankle joints after forward and lateral drop landing in healthy young women. Isokinet Exerc Sci. 2019;27:1-7.

6. Castro A, Goethel MF, Gáspari AF, Crozara LF, Gonçalves M. Ankle brace attenuates the medial-lateral ground reaction force during basketball rebound jump. Rev Bras de Medicina do Esporte. 2017;23:232-6.

7. Henderson Z, Sanzo P, Zerpa C. The effect of prophylactic ankle bracing on physical performance measures in jumping athletes. Int J Sports Sci. 2016;6:138-45.

8. Henderson ZJ, Sanzo P, Zerpa C, Kivi D. Ankle bracing's effects on lower extremity iEMG activity, force production, and jump height during a Vertical Jump Test: An exploratory study. Phys Ther Sport. 2019;37:171-8.

9. Henderson ZJ, Sanzo P, Zerpa C, Kivi D. The effects of ankle braces on lower extremity electromyography and performance during vertical jumping: a pilot study. Int J Exerc Sci. 2019;12:15-23. 
10. Mann B, Gruber AH, Murphy SP, Docherty CL. The influence of ankle braces on functional performance tests and ankle joint range of motion. J Sport Rehabil. 2019;28:817-23.

11. Smith B, Claiborne T, Liberi V. Ankle bracing decreases vertical jump height and alters lower extremity kinematics. Int J Athl Ther Train. 2016;21:39-46.

12. You DZ, Tomlinson M, Borschneck G, Borschneck A, MacDonald M, Deluzio K, et al. The effect of ankle brace use on a 3-step volleyball spike jump height. Arthrosc Sports Med Rehabil. 2020;2:e4617.

13. Nariai M, Yoshida N, Imai A, Ae K, Ogaki R, Suhara H, et al. A biomechanical comparison among three kinds of rebound-type jumps in female collegiate athletes. Int J Sports Phys Ther. 2017;12:560-8.

14. Healy R, Kenny IC, Harrison AJ. Reactive strength index: a poor indicator of reactive strength? Int J Sports Physio Perform. 2018;13:802-9.

15. Barker LA, Harry JR, Mercer JA. Relationships between countermovement jump ground reaction forces and jump height, reactive strength index, and jump time. J Strength Cond Res. 2018;32:24854.

16. Pescatello LS, Riebe D, Thompson PD, editors. ACSM's Guidelines for Exercise Testing and Prescription. 9th ed. Philadelphia: Wolters Kluwer/Lippincott Williams and Wilkins Health; 2014.

17. Comyns TM, Flanagan EP, Fleming S, Fitzgerald E, Harper DJ. Interday reliability and usefulness of a reactive strength index derived from 2 maximal rebound jump tests. Int J Sports Physiol Perform. 2019;1200-4.

18. Yamauchi J, Koyama K. Importance of toe flexor strength in vertical jump performance. J Biomech. 2020;104:109719.

19. Gruber M, Kramer A, Mulder E, Rittweger J. The importance of impact loading and the stretch shortening cycle for spaceflight countermeasures. Front Physiol. 2019;10:311.

20. Flanagan EP, Comyns TM. The use of contact time and the reactive strength index to optimize fast stretch-shortening cycle training. Strength Cond J. 2008;30:32-8.

21. McBride JM, Kirby TJ, Haines TL, Skinner J. Relationship between relative net vertical impulse and jump height in jump squats performed to various squat depths and with various loads. Int J Sports Physiol Perform. 2010;5:484-96.

\section{Tables}

Due to technical limitations, table 1 is only available as a download in the Supplemental Files section.

\section{Figures}




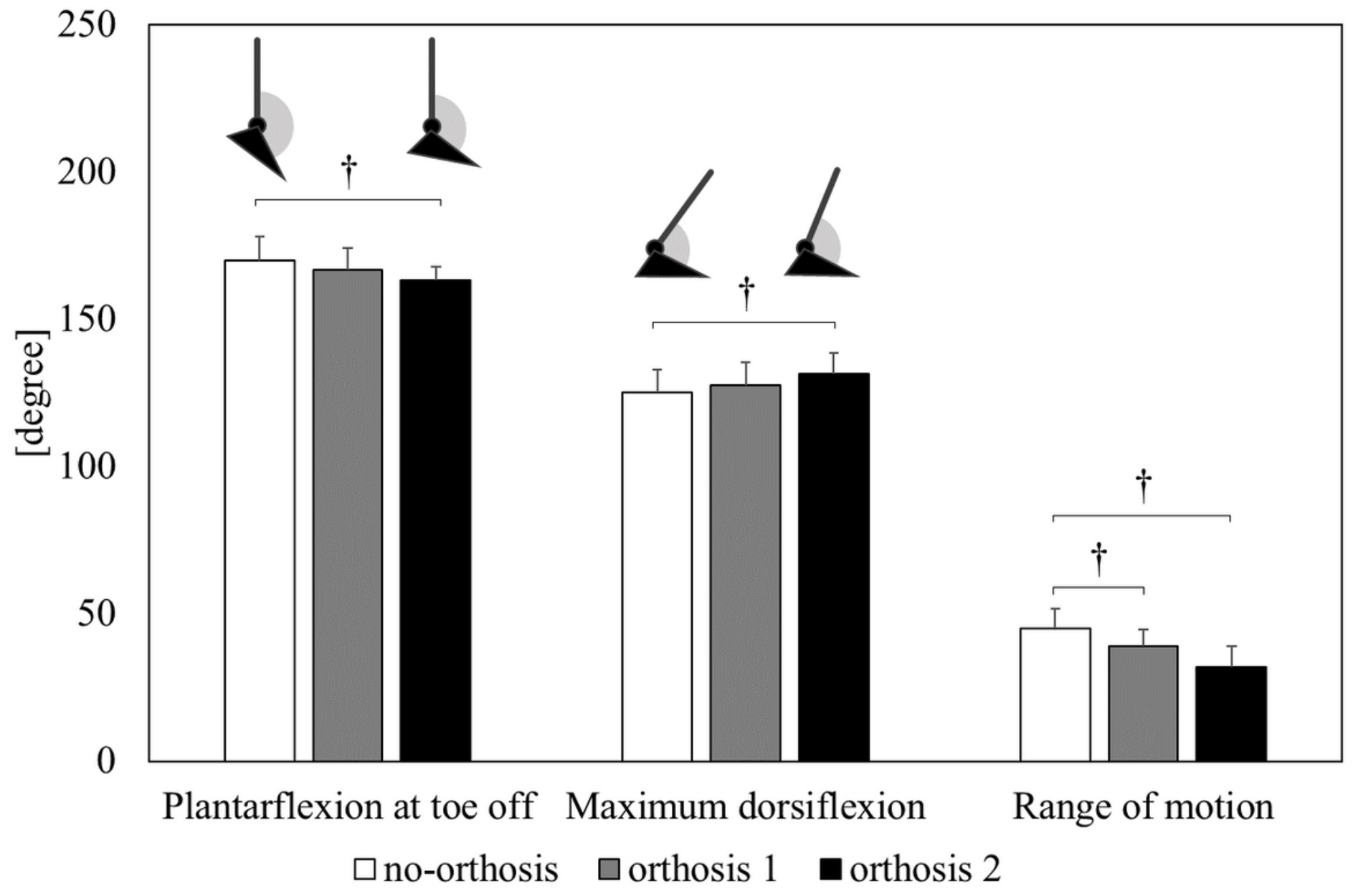

Figure 1

Ankle orthotics used in this study. A: The long-dotted line indicates the hard film in the surface layer. The short-dotted line indicates the soft film in the middle layer. B: The solid line indicates the stirrup strap. The long-dotted line indicates the biceps strap. The short-dotted line indicates the strap for distal tibiofibular joints.
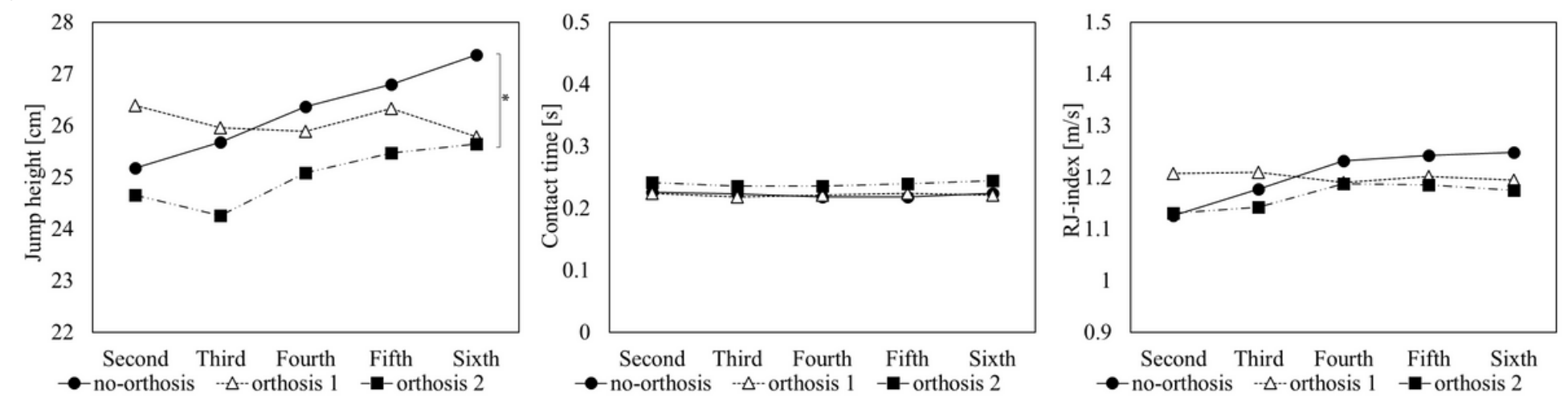

Figure 2

Video analysis of maximum dorsiflexion angle (A), plantarflexion at toe-off (B), and dorsi-plantarflexion ROM (C). ROM, range of motion 

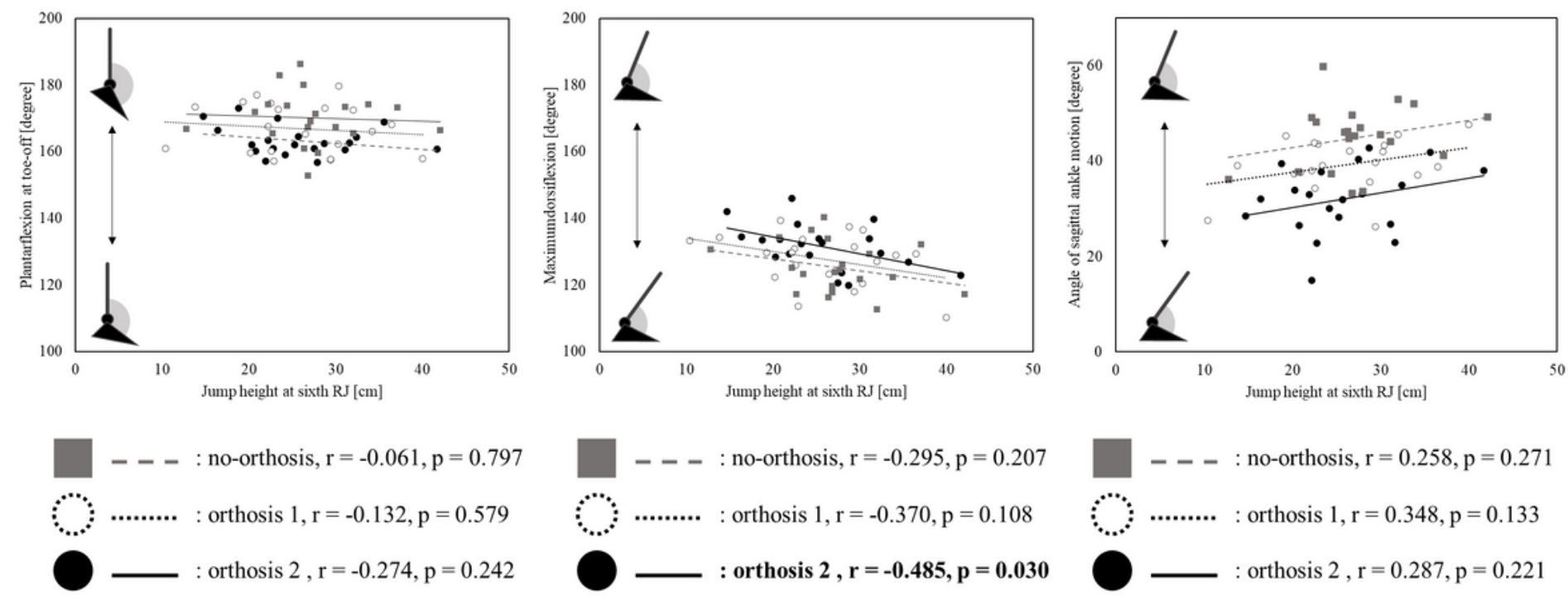

- - $\quad$ : no-orthosis, $r=0.258, p=0.271$

:......... : orthosis $1, r=0.348, p=0.133$

- orthosis $2, r=0.287, p=0.221$

\section{Figure 3}

Impact of two types of ankle orthotics on repetitive rebound jump performance. Two-factor (condition: no-orthosis, orthosis 1 , orthosis $2 \times$ number of rebound jumps: second, third, fourth, fifth, sixth) repeatedmeasures multivariate analysis of variance showed significant difference in repetitive rebound jump performance $(f=7.020, p<0.001)$. One-factor (condition: no-orthosis, orthosis 1 , orthosis 2 ) repeatedmeasures analysis of variance and pairwise comparisons revealed a significant decrease in jump height in orthosis 2 compared to the no-orthosis condition. $\dagger=$ statistically significant difference $(p<0.01)$.

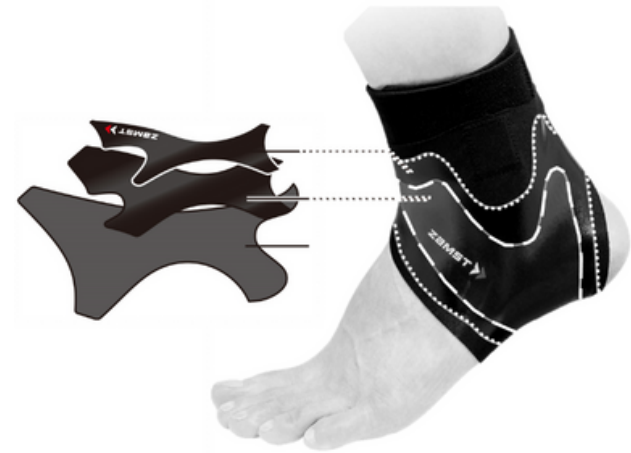

A: orthosis 1 (Filmista Ankle)
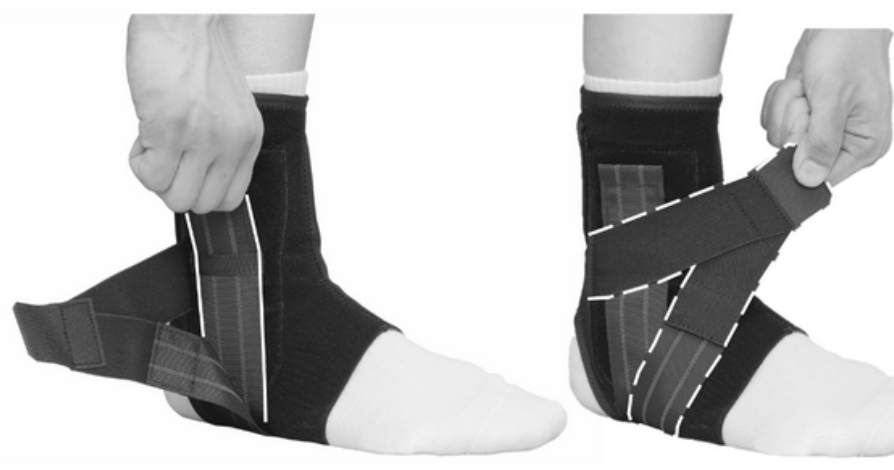

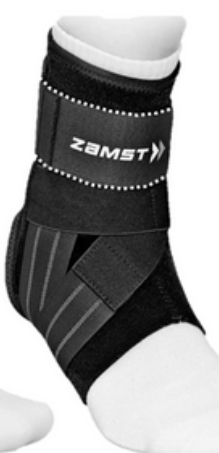

B: orthosis 2 (A1)

Figure 4

Influence of two types of ankle orthotics on sagittal angle of ankle joint at the sixth rebound jump. $\dagger=$ statistically significant difference $(p<0.01)$ 


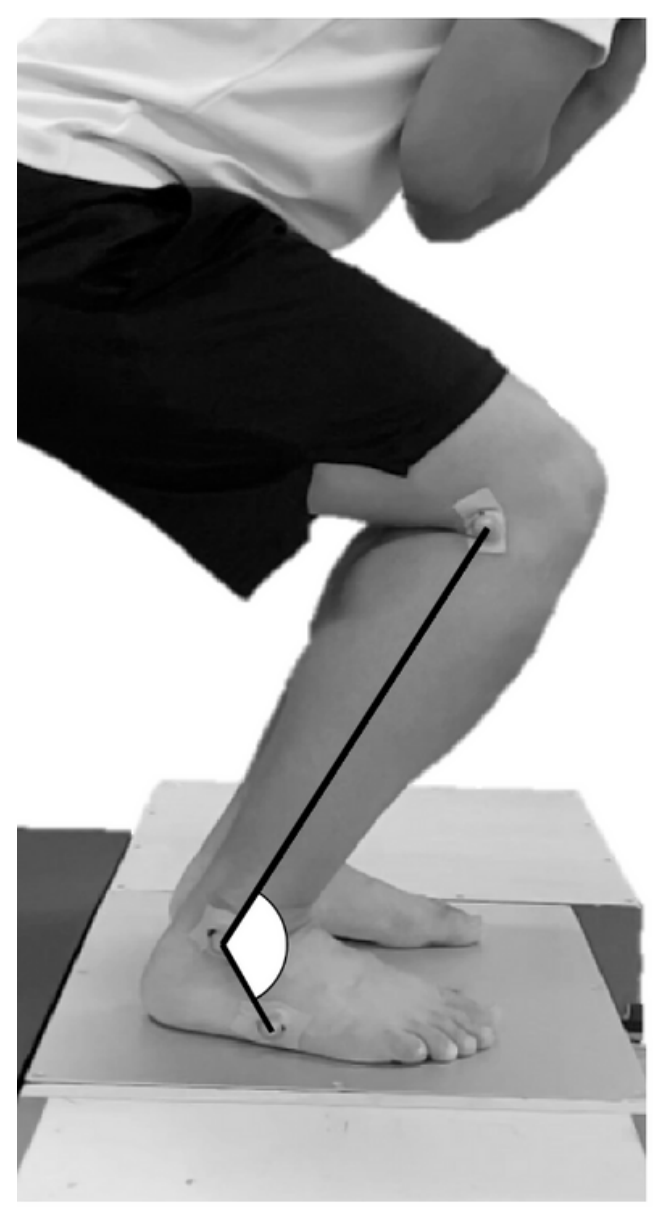

A: Maximum dorsiflexion angle

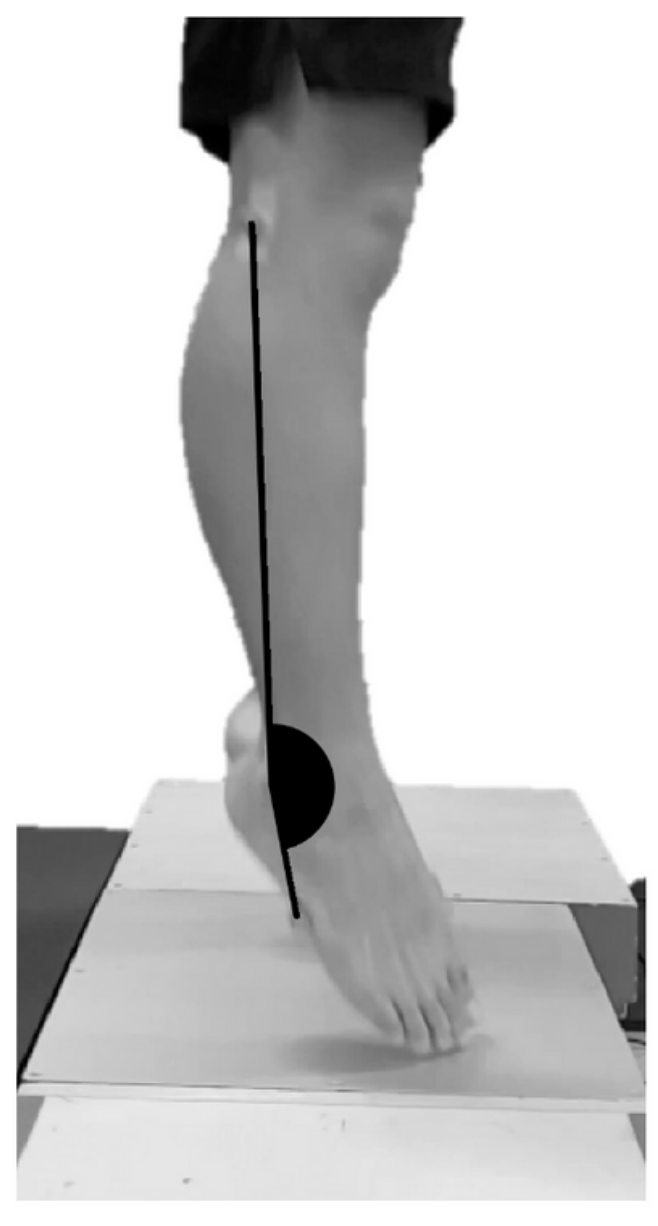

B: Plantarflexion angle at toe off

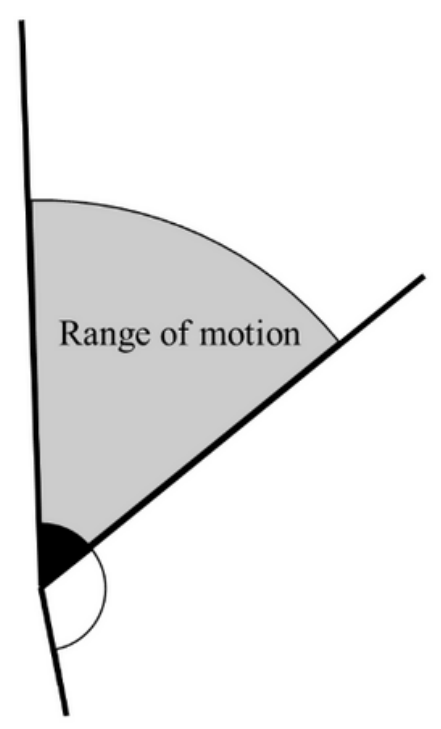

\section{Figure 5}

Relationship between rebound jump performance and the angle of sagittal ankle motion at the sixth rebound jump in orthotic conditions.

\section{Supplementary Files}

This is a list of supplementary files associated with this preprint. Click to download.

- Table1.xlsx 